\title{
Cardiac troponin elevation as an evidence of neurogenic myocardial dysfunction in patients with head trauma
}

\section{Prof.Abdelrahman Hassan Abdelrahman, Dr.Wesam Abdgalil, Al haitham Mohamed Taha}

\section{Abstract}

Background: cardiac dysfunction is frequently observed after severe traumatic brain injury (sTBI), however its significance is poorly understood. Our study sought to elucidate the association of cardiac troponin $\mathrm{I}(\mathrm{cTnI})$ elevation with all cause in-hospital mortality following isolated sTBI (brain AIS $\geq 3$ and admission GCS $\leq 8$, no AIS $\geq 3$ to any other bodily regions).

Patients and methods: After approval of this study from local ethical committee, and obtaining written informed consent from relatives, head trauma patients will be enrolled in this observational prospective study. study was conducted in the 10-bed trauma-surgical ICU in Sohag university hospital, Fifty patients with isolated TBI, were consecutively included between January 2015 and February 2017.

Results: our study validated cTnI as a novel biomarker and independent predictor of all cause in-hospital mortality in patients with TBI. these findings have several implications. First, cTnI assay should be considered in patients with sTBI even in absence high suspicion of cardiac injury. Second, cTnI is a sufficiently prognostic biomarker of mortality in patients with sTBI. Third, cTnI elevation in patients with sTBI must be treated with caution.

Conclusion: cTnI level was a significant indicator for stress cardiomyopathy , severity of trauma calculated by RTS had no relation with incidence of stress cardiomyopath . Our study provided important insights to the heart-brain interactions following TBI and possible schemes for subsequent optimization of management of these patients.

\section{Introduction:-}

Cardiac troponin is a regulatory protein that regulates the calcium mediated interaction of actin and myosin. It is used as a more sensitive and specific biochemical marker of myocardial injury than creatine kinase (1).

Besides its use in diagnosing acute coronary syndromes, cardiac troponin elevation has been observed in a variety of non-coronary conditions such as pulmonary embolism, sepsis, and chronic renal failure in the absence of acute myocardial infarction ${ }^{(2),(11)}$.

Troponin elevation has also been observed in acute non traumatic head injury including subarachnoid hemorrhage, stroke, and intracerebral hemorrhage (3), (4), (13). The cause of troponin elevation after these neurologic events is not clear, but may be due to increased circulating levels of catecholamines after brain injury ${ }^{(5)}$. Elevation of troponin after non traumatic cerebral insult may help to predict patients outcome. It has been associated with an increased risk of cardiopulmonary complications and mortality ${ }^{(6),(7)}$.

Previous studies examining the role of troponin in trauma have focused on blunt thoracic trauma and blunt cardiac injury (8), (9), (12). Association between traumatic brain injury and troponin elevation has not been well studied. Few studies discussed this issue ${ }^{(8),(10)}$. In this study, we will report incidence of elevated Cardiac troponin in patients with head trauma, and identify this as an evidence of myocardial dysfunction. 
SOHAG MEDICAL JOURNAL Cardiac troponin elevation as an evidence of neurogenic myocardial dysfunction

Aim of the study:-

- Report incidence of elevated cardiac troponin in patients with head trauma.

- Identify elevated cardiac troponin as an evidence of transient neurogenic myocardial dysfunction.

Patients and methods:

After approval of this study from local ethical committee, and obtaining written informed consent from relatives, head trauma patients will be enrolled in this observational prospective study. study was conducted in the 10-bed traumasurgical ICU in Sohag university hospital, Fifty patients with isolated TBI , were consecutively included between January 2015 and February 2017.

Criteria we excluded from our study:

- Patient with history of hypertension.

- Patient with history of smoking.

- Patient with history of D.M.

- Patient with history of congenital heart disease.

- Patient with history of dyslipidemia.

- Age above $30 \mathrm{y}$.

- Age below $12 \mathrm{y}$.

- Patient with family history of cardiac disease.

- Patient with associated heart or chest trauma.

- Female Pt. under oral contraceptive pills usage.

Upon admission to ICU, patients' GCS ( Glasgo coma scale ), Temperature, non-invasive BP , fivelead electrocardiography, hourly urinary output and central venous pressure (CVP) were monitored in all patients.

Endotracheal intubation and mechanical ventilation were performed to protect airway and maintain $\mathrm{PaCO} 2$ between 35 and $40 \mathrm{mmHg}$.
Electrocardiogram (ECG) were monitored every 2 hours; ECG abnormalities in the form of ( supraventricular arrythmias, STsegment deviation, T-wave inversion, ventricular ectopics ) were evaluated. In patients with an abnormal ECG, cardiac Troponin was assessed .

Our laboratory reference range for Troponin value defines a normal cardiac Troponin between ( $0-0.3$ $\mathrm{ng} / \mathrm{mL}$ ); Values more than ( 0.3 $\mathrm{ng} / \mathrm{mL}$ ) was considered abnormal. In patients with elevated cardiac Troponin values, transthoracic echocardiography was performed to identify apical pallooning and/or regional wall motion abnormalities.

Collected demographic and clinical information were included : age, gender, type and site of the lesion.

GCS was recorded every day until patient death or discharge from ICU; For patients who needed sedation, GCS was assessed 3-4 h after stoppage of sedation, Sedation was done by using Midazolam (20-100 mcg/kg/hr) and/or Fentanyl (1-2 mcg/kg/hr).

Also, we depend upon 2 scores for patient evaluation in our study; Revised trauma score and Marshall CT score. We choose RTS because it is heavily weighted towards the Glasgow Coma Scale to compensate for major head injury without multisystem injury or major physiological changes.

Revised trauma score (RTS) was assessed and calculated for all patients on admission as follows: RVS is made up of three parameters: Glasgow Coma Scale, Systolic blood pressure, and respiratory rate; these Parameters are then used to take the weighted sum by $\mathrm{RTS}=(0.9368) \mathrm{GCS}+(0.7326) \mathrm{SBP}+$ (0.2908) RR. Values for the RTS are in the range $(0-7.8408)$. A threshold of RTS $<4$ has been proposed to identify those patients who should be managed as severe trauma patients. 
SOHAG MEDICAL JOURNAL Cardiac troponin elevation as an evidence of neurogenic myocardial dysfunction

Routine head CT head computed tomography (CT), then Marshall CT score was assessed ,The Marshall classification of traumatic brain injury is a CT scan derived metric using only a few features and has been shown to predict outcome in patients with TBI.

\section{Results:-}

Eighty patients were admitted to our ICU with TBI within a period of 48 months from March 2015 to March 2017, Ninety-four patients had fullfiled our criteria and were enrolled in the study.

15 patients were excluded being above 30 years old, three patients were excluded due to cardiac arrest shortly after ICU admission, seven patients were excluded due to associated chest trauma and six patients were excluded due to previous history of smoking.

Demographic data for our patients were shown in table :

\begin{tabular}{|l|l|l|l|}
\hline & Range & Mean & Std. Deviation \\
\hline Age (y) & $(8-30)$ y & 23.0408 & 6.2648 \\
\hline Weight $(\mathrm{kg})$ & $(25-95) \mathrm{kg}$ & 69.8367 & 14.6988 \\
\hline Height $(\mathrm{cm})$ & $(110-190) \mathrm{cm}$ & 169.7551 & 15.3100 \\
\hline
\end{tabular}

Table (1) Patient's characteristics

All elligable patients were included in the final analysis ( 49 patients ); 47 (95.9\%) of them being males and $2(4.1 \%)$ females with a mean age of (23.04), mean weight of ( 69.83 ) and with a mean height

$(169.75)$.

Patients with isolated head trauma were 34 (69.4\%); while patients with head trauma and other body trauma were $15(30.6 \%)$ in the form of ; facial bone fractures in one patient $(2 \%)$, long bone fractures in two patients $(4.1 \%)$, fracture arm in one patient (2\%) and poly traumatized patients were seven $(14.3 \%)$.

Revised trauma score (RTS) was calculated; mean value was (4.7592) and standard

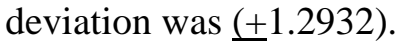

\begin{tabular}{|l|l|l|l|l|l|}
\hline Number & Mean & Median & SD & Minimum & Maximum \\
\hline 49 & 4.7592 & 4.7000 & 1.2932 & 2.30 & 7.80 \\
\hline
\end{tabular}

Table (2) Revised trauma score

Patients with RTS less than the median RTS value 4 were 14 patients $(28.5 \%)$ while patients with median RTS more than 4 were 35 patients $(71.5 \%)$.

Patients discharged from ICU to the ward after complete improvements were 33 patients $(67.3 \%)$.

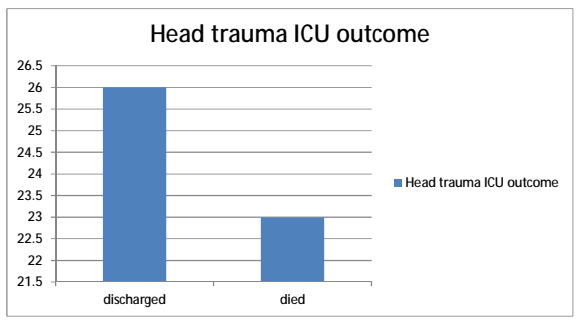

Fig ( 1 ) head trauma ICU outcome 
SOHAG MEDICAL JOURNAL Cardiac troponin elevation as an evidence of neurogenic myocardial dysfunction

Patients with GCS less than 7 were 33 patients (67.3\%) with severe head trauma, while patients with GCS more than 7 were 16 patients (32.7\%).

Surgical intervention were done for 15 patients; elevation of depressed skull fracture in one patient $(2 \%)$, evacuation of extradural hematomas in three patients (6.1\%), fixation of femur fractures in two patients $(4.1 \%)$, fixation of fracture humerus in one patient $(2 \%)$ and surgical intervention of poly traumatized patients with surgical exploration and fixation of bone fractures were done for seven patients $(14.3 \%)$.

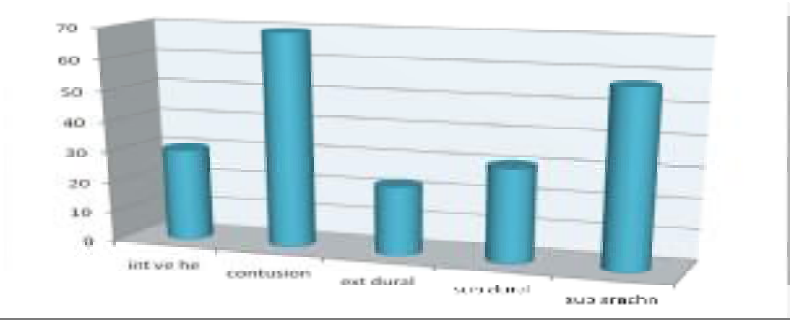

Fig (2) Types of brain injury

The primary traumatic brain lesions were as follows: Inter ventricular hemorrhage in 15 patients $(30.6 \%)$, brain contusions in 34 patients $(69.4 \%)$, extradural hemorrhage in 11 patients $(22.4 \%)$, subdural hemorrhage in 15 patients $(30.6 \%)$ and subarachnoid hemorrhage in 28 patients $(57.1 \%)$.

Also, Marshall CT score was calculated to patients in our study and the results were as follow:

\begin{tabular}{|l|l|l|l|l|l|l|}
\hline CT score & CT1 & CT2 & CT3 & CT4 & CT5 & CT6 \\
\hline No. of patients & 1 & 19 & 23 & 4 & 1 & 1 \\
\hline percentage & $2 \%$ & $38.8 \%$ & $46.9 \%$ & $10.2 \%$ & $2 \%$ & $2 \%$ \\
\hline
\end{tabular}

Table (3) Marshall CT score results

Patients with sever TBI (Traumatic Brain Injury) were 29 patients (59.2\%), while patients with mild to moderate TBI were 20 patients $(40.8 \%)$.

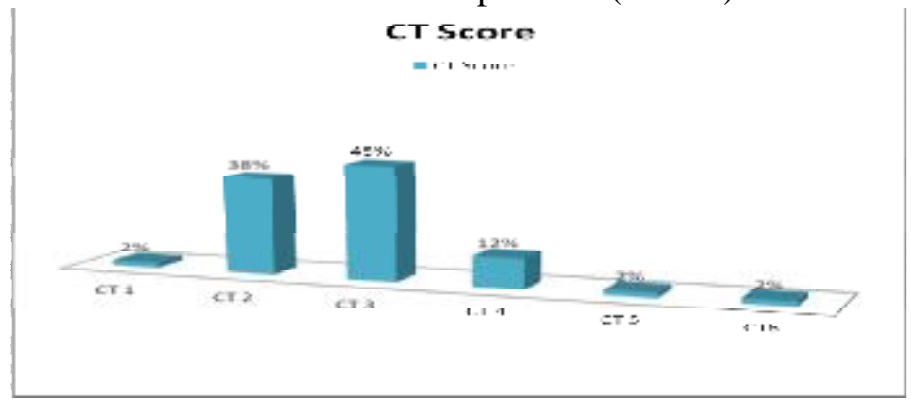

Fig (3) Marshall CT score

Cardiac assessment for our patients revealed that; among the 49 patients, 17 patients (34.7\%) developed hypotension and needed inotropes infusion; 4 patients $(8.2 \%)$ received Dopamine in the first day and continued with Dopamine infusion by rate of $(0.1-0.5 \mathrm{mcg} / \mathrm{kg} / \mathrm{min})$ till discharge. The other 13 patients $(26.5 \%)$ received Dopamine infusion from the first day by the same rate and stopped within 48 hours. The remaining 32 patients (65\%) did not need any inotropes since admission till discharge. ECG values showed abnormalities in 11 patients $(22.4 \%)$ at the first day of admission in the form of ; two patients $(18.1 \%$ ) had ventricular ectopics, three patients $(27.2 \%)$ developed supraventricular tachycardia and nine patients $(81.8 \%$ ) had significant ST segment elevation. 
SOHAG MEDICAL JOURNAL Cardiac troponin elevation as an evidence of neurogenic myocardial dysfunction

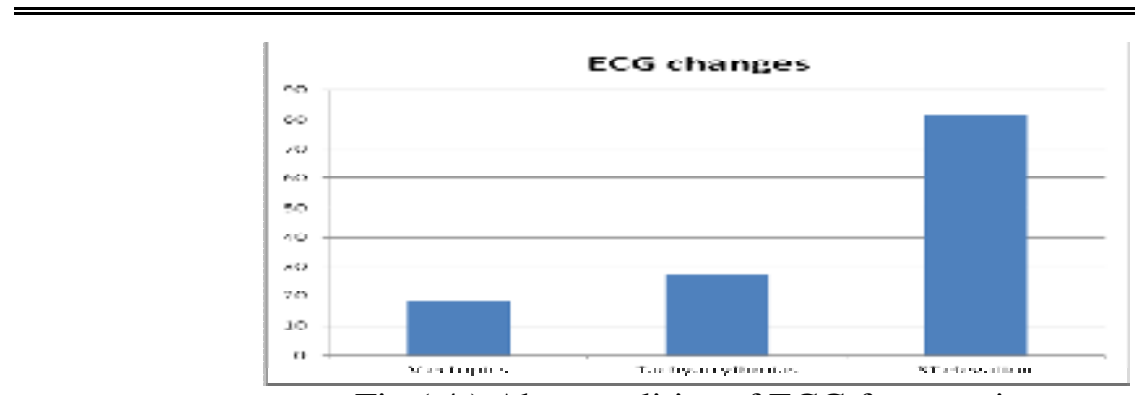

Fig ( 4 ) Abnormalities of ECG frequencies

All patients who developed ECG changes (11 patients) were tested for ( Troponin I ) level ; nine patients $(18.36 \%)$ had elevated troponin level while two patients $(4.08 \%)$ showed normal troponin level even after follow up assessment.

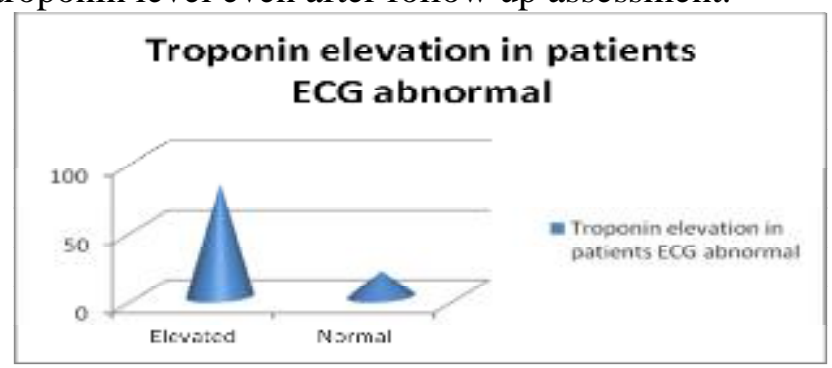

Fig ( 5 ) Troponin elevation in patients with ECG abnormalities

All ( 9 patients ) with elevated ( Troponin I ) level had subjected to Echocardiographic assessment which showed cardiac dysfunction in nine patients $(18.36 \%)$ in the form of ; apical ballooning in six patients $(12.2 \%)$ and wall motion abnormalities in three patients $(6.12 \%)$.

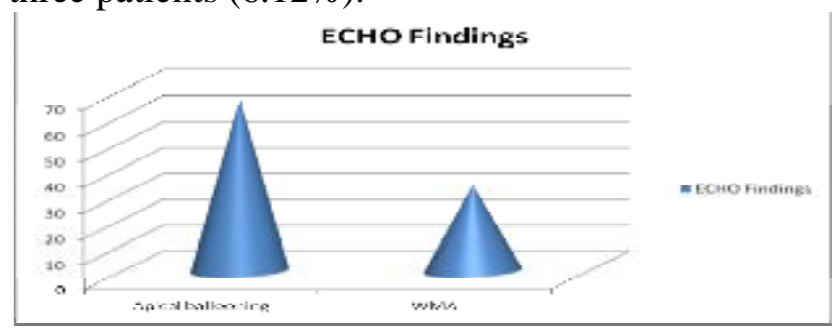

Fig (6) Echocardiography findings in elevated Troponin Pts.

As regard the relation of troponin level to the type of head trauma lesion, we founded that six patients $(66.6 \%)$ with elevated troponin level had subarachnoid hemorrhage, one patient $(11.1 \%)$ had extradural hemorrhage and two patients $(22.2 \%)$ had brain contusions.

Also, as regard the relation between the Revised trauma score and the Troponin level in our patients we found that; five patients with elevated troponin level $(55.5 \%)$ had Revised trauma score $>4$ while four patients $(45.5 \%)$ were $<4$.

Two patients (22.2\%) were discharged after complete recovery.

\section{Discussion:-}

The "gold standard" for acute myocardial damage, Troponin is a highly sensitive and specific biomarker. In addition to acute coronary syndrome, Troponin elevation has also been observed following a variety of noncoronary conditions such as pulmonary embolism, pulmonary hypertension, sepsis, and chronic renal failure and non-traumatic head injuries such as sub arachnoid haemorrhage, inter ventricular haemorrhage, acute stroke, seizure, and Guillain-Barre syndrome. In these settings, particularly SAH 
SOHAG MEDICAL JOURNAL Cardiac troponin elevation as an evidence of neurogenic myocardial dysfunction

elevated cardiac Troponin I was associated with increased risk of cardiopulmonary and cerebrovascular complications, as well as increased mortality and worse functional outcomes. ${ }^{(14,15)}$ Pathophysiological

mechanism of neurogenic stunned myocardium is poorly understood however, the prevailing theory is related to a systemic catecholamine surge driven by the central neuroendocrine axis which massively increases sympathetic outflow and activates the adrenal glands. Damage to the insular and hypothalamus also initiates a complex cascade of events, including activation followed by dysfunction of the autonomic nervous system and an acute inflammatory response, which may incur major adverse effects on the heart. ${ }^{(13)}$

Brain injury-related ECG abnormalities have been recognized for more than five decades and are particularly common after SAH where they are reported in $49-100 \%$ of cases. The most common findings are ST segment changes, flat or inverted $\mathrm{T}$ waves, prominent $\mathrm{U}$ waves, and prolongation of the QT interval (QT interval corrected for heart rate). Clinical studies showed no correlation between circulating catecholamine levels and ECG abnormalities, suggesting that they are also due to myocardial injury secondary to local sympathetic activation. ${ }^{(21)}$

Similar phenomenon was thought to occur following TBI. Several studies were able to quantify an increase in sympathetic activity after TBI by measuring plasma and urinary catecholamine levels. ${ }^{(16,17)}$ Furthermore, beta-blocker therapy has shown to confer a survival advantage to TBI patients with elevated cardiac enzymes. ${ }^{(19,20)}$ Despite growing evidence that suggested a potential link between cardiac dysfunction and TBI, only few studies have investigated the clinical outcomes of patients with cardiac dysfunction and TBI.

In our study we aim to identify the relation between head trauma and the occurring of stress cardiomyopathy and using of cardiac troponin I as indicator of cardiac dysfunction.

In our study the mean age was $(23 \pm 6$ years) with exclusion of other risk factors of ischaemic heart diseases, however in other studies the mean age of patients under their study was higher, in Prathep's study was (58 \pm 20 years) (25), the age of patients studied in Hasanin et al. ${ }^{(24)}$ was $(31 \pm 12$ years $)$ with age limit 50 years old.

In the current study eleven patients with TBI $(22.4 \%)$ developed cardiac injury as documented by abnormal ECG , in the form of ; two patients ( $4.08 \%$ ) had ventricular ectopics, three patients ( 6.1 $\%$ ) developed supraventricular tachycardia and nine patients ( $18.3 \%$ ) had significant ST segment elevation, unlike ECG findings recorded by Chadi etal and his colleague Of the 105 patients identified with head trauma, 35 patients $(33 \%)$ had ST-segment elevation on the ECG at admission and 1 patient $(0.9 \%)$ had new onset of LBBB, 32 patients $(30 \%)$ had T-wave inversions and 34 patients $(32 \%)$ had nonspecific ST segment elevation, ${ }^{(22)}$ that difference may be attributed to many factors, first there was no age limit in his study with mean age 69 years old, they did not exclude patients with IHD or chest trauma.

Vijay ,etal and his colleagues studied 59 patients with head trauma they found ECG abnormalities in 12 pt. (20.3\%) In the form of Prolonged PR interval occurred in $(6.8 \%)$ of patients, while a prolonged QTc interval was diagnosed in $(42.4 \%)$ of patients. Ischemic-like ST-elevation $(23.5 \%)$ and $13(22 \%)$ patients had supra ventricular tachycardia, ${ }^{(23)}$ these results in line with our study as regard they exclude 
SOHAG MEDICAL JOURNAL Cardiac troponin elevation as an evidence of neurogenic myocardial dysfunction

patients with IHD and patients with chest trauma.

Hasanin et al. ${ }^{(24)}$ in his study reported that cardiac assessment of patients revealed that among the 50 patients, the ECG for $31(62 \%)$ patients showed abnormalities, six (12\%) patients had abnormal QT interval and $29(50 \%)$ of patients developed tachyarrhythmia, the higher incidence of ECG changes in his study may be attributed to higher mean age of their patients, and they didn't exclude patients with heart and chest trauma, and patients with risk factors for IHD.

In the current study elevated Troponin I was found in nine patients $(18.36 \%)$, that gave us strong indicator ECG changes and elevation of cardiac Troponin I in young head trauma patients should considered having cardiac dysfunction until proved otherwise.

The incidence of cardiac dysfunction reported by Prathep and colleagues in patients with TBI is near in line with our results. They reported elevated serum troponin in $24 \%$ of patients, our results were not in line with Prathep's study ${ }^{(25)}$ however they were defected in ; no data about patients' ECG abnormalities, also our patients had a mean age of $23 \pm 6$ years which is generally lower than the patients included in Prathep's study ${ }^{(25)}(58 \pm 20$ years).

Hasanin et al. (24) in his study reported that half of the patients developed cardiac injury as documented by elevated Troponin I in $54 \%$ of patients. The incidence of cardiac injury in our study $18.3 \%$ documented by elevated Troponin I , This difference might be due to many factors. First, the age limits in Hasanin et al. ${ }^{(24)}$ was 50 years old compared to in our study 30 years old also, in their study they didn't exclude patients with IHD. Second all patients included in Hasanin et al. ${ }^{(24)}$ had severe TBI. In contrast, only $59 \%$ in our study were diagnosed with severe TBI.

Serri et al. ${ }^{(26)}$ recently reported a low incidence of cardiac injury among 41 patients with severe TBI. Serri's findings differed from our reports as well as Prethep's findings, this difference warrants more studies with larger sample size to investigate the actual incidence of cardiac injury in TBI.

Echocardiographic assessment was done for all patients who showed ECG changes in our study we found cardiac dysfunction in nine patients of them $(18.36 \%)$ in the form of ; six patients (12.2\%) had apical ballooning and three patients $(6.12 \%)$ with wall motion abnormalities .

Prathep et al reported abnormal echocardiogram, including reduced left ventricular ejection fraction and regional wall motion abnormality, were present in $22.3 \%$ of patients with TBI . ${ }^{(25)}$ Similarly, Hasanin et al. ${ }^{(24)}$ in his study reported that echocardiographic examination revealed abnormalities in $14(28 \%)$ patients, five $(10 \%)$ of them were apical ballooning and (14\%) were presented by segmental wall motion abnormalities.

In the current study, we reported an increased incidence of in-hospital mortality in TBI patients with cardiac injury. Consistent with our findings, cardiac injury was a mortality risk factor in patients with TBI ${ }^{(25)}$, CVS ${ }^{(27,28)}$, and SAH ${ }^{(29,30)}$. Some authors reported cardiac injury to be a reversible condition that improves over time ${ }^{(31)}$

Finally all previous studies did not use any of the Trauma scores or Imaging scores, they did not mention types and site of head injury which is directly connected with the severity of cardiac injury and patients outcome.

In our study we used Revised Trauma Score (RTS) which is heavily weighted towards head injuries and Marshall CT 
SOHAG MEDICAL JOURNAL Cardiac troponin elevation as an evidence of neurogenic myocardial dysfunction

score which directly affected by the size and site of the lesion which showed us better idea for outcome.

Computed tomography (CT) plays a crucial role in early assessment of patients with traumatic brain injury (TBI). Marshall and Rotterdam are the mostly used scoring systems, in which CT findings are grouped differently. We sought to determine the scoring system and initial CT findings predicting the death at hospital discharge (early death) in patients with TBI.

Both Marshall and Rotterdam scoring systems can be used to predict early death in patients with TBI. The performance of the Marshall score is at least equal to that of the Rotterdam score. Thus, although older, the Marshall score remains useful in predicting patients' prognosis.

In our study we use Marshall CT score and our results revealed that, Patients with severe TBI (Traumatic Brain Injury) with Marshall CT scores ( CT4, CT5, CT6) were 6 patients $(14.1 \%)$, while patients with moderate TBI (CT3) were 23 patients $(46.9 \%)$, and patients with mild TBI (CT1, CT2) were 20 patients $(40.8 \%)$.

We would like to highlight the strength of our study. Most previous studies assessing CT scores in patients with TBI included only moderate and/or severe cases $(32,33,34,35,36,39,40,41)$. We presume that the authors excluded mild cases because the main objective of most studies was to assess long-term mortality and functional outcomes after TBI (42) and because they were concerned that the inclusion of mild cases, many of whom might have not undergone CT (possibly with resultant selection bias), would increase the number of clinically insignificant cases in their series. In contrast to previous studies, they included a considerable number of patients with mild TBI who underwent CT examination according to the New Orleans criteria and/or Canadian CT were considered mild at admission. This result is consistent with that reported by Jacob et al. ${ }^{(43)}$ who also evaluated the prognostic power of CT findings in patients with mild TBI whose CT examination was conducted according to some specific clinical criteria.

Finally their data showed that Marshall scoring systems can be used to predict early death in patients with TBI. The performance of Marshall scoring system is equal or possibly slightly better than that of Rotterdam scoring system. This statement is supported by our specific results showing that the two strongest predictors of early death-basal cistern absence and positive midline shift — are included in both CT scoring systems, but voluminous hemorrhagic mass, the next strongest predictor, is included only in Marshall score. Thus, although older than Rotterdam score, Marshall score remains useful for the purpose of outcome prediction patients $\frac{(47)}{}$, and another at the severe (trauma score $<6$ ) end of the injury spectrum $\stackrel{(64)}{\text {, found }}$ no association between the RTS and mortality. These studies potentially failed to identify an association between mortality and the RTS due to the homogeneity of the study population and/or insufficient power to detect the relationship.

Other studies have focused on the ability of the RTS to predict morbidity ${ }^{(49)}$, disability ${ }^{(47,51,52)}$, ISS $15^{(44,45)}$, length of hospital stay ${ }^{(49)}$, and intensive care unit admission $\stackrel{(44)}{ }$, with varying results. Gray al et. assessed the accuracy of the RTS for identifying patients with 'major trauma' in a small population ( $n 213)$ of trauma patients in the UK $\stackrel{(44)}{ }$. In that study, major trauma was defined as ISS $>15$, intensive care unit admission and/or death after arrival in the emergency 
SOHAG MEDICAL JOURNAL Cardiac troponin elevation as an evidence of neurogenic myocardial dysfunction

department $\stackrel{(44)}{ }$. At a cut-off of RTS $<12$, the sensitivity was 0.60 and the specificity was 0.90 . The relatively low sensitivity suggests that use of the RTS alone will lead to under-triage of major trauma patients. However, the small, retrospective nature of the study must be considered when interpreting the results. For the study, the raw RTS was used. Whether the weighted RTS would have provided different results cannot be determined.

As regards RTS, the present study showed that the mean RTS score was $(4.51 \pm 1.46)$ in the poor prognosis group, while in good prognosis group the mean RTS was $(5.74 \pm 1.05)$, there was a significant increase in RTS in good prognosis group than poor prognosis group $(\mathrm{p}<0.01)$. Our findings were Patients with RTS less than the median RTS value were 14 patients $(28.5 \%)$ while patients with more than RTS median value were 35 patients $(71.5 \%)$.

Patients discharged from ICU to the ward after complete improvements were 33 patients $(67.3 \%)$, these findings are similar to Hafiz ${ }^{(53)}$ study in 2004 conducted at Nishtar Hospital Multan From August 1999 to January2001.He studied 30 adult patients of road traffic accidents sustaining multisystem injuries due to high energy blunt trauma and were managed according to the protocols of advanced trauma life support (ATLS) and from their first set of data RTS was calculated. Score of each patient was compared with his final outcome at the time of discharge from the hospital. He found that RTS is a reliable predictor of prognosis of polytraumatized patients. Therefore, it can be used for field and emergency room triage. Also, Ohaegbulam et $\mathrm{al}^{(54)}$, conducted a prospective study on relationship between the weighted revised trauma score and patient outcome (mortality),
The records of 38 critically injured trauma patients admitted to the general ICU of National Hospital, Abuja, Nigeria over a nine-month period (April - December 2005) were analyzed. The results confirmed that RTS is a good predictor of both severity of head injury (and thus the need for ICU admission) and mortality.

such, a number of studies have investigated whether the RTS can predict the level of disability or morbidity following traumatic injury As techniques for trauma management have evolved and the quality of patient care improves, the likelihood of surviving even severe traumatic injuries has increased. This has led to an improved $\mathrm{As}^{(47,49,51,52)}$.Moini al et., in their prospective study of 2662 trauma patients in Iran found no association $(P$ $>0.05$ ) between the RTS and morbidity

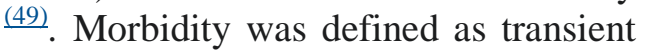
or permanent disability, but no detail regarding the measurement of this outcome was provided. ${ }^{(47,51)}$.

Zafonte al et. collected data prospectively for 501 patients with traumatic brain injury in the USA $\stackrel{(51)}{\text {. }}$ The RTS was recorded on admission to the emergency department and was then compared with the patient's performance on the disability rating scale (DRS) at both rehabilitation admission and discharge $\frac{(51)}{}$. A significant but only modest correlation was found between the RTS and the DRS scores, suggesting 120 patients who sustained a traumatic brain

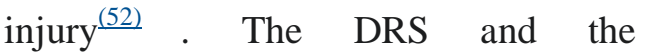
Community Integration Questionnaire were used to evaluate disability. The RTS was significantly associated with both the DRS and the Community Integration Questionnaire. Patients with an RTS<times more likely to record a poor DRS score ( 9 were $6.3 P$ $<0.01$ ), and the RTS was also an independent predictor of the 
SOHAG MEDICAL JOURNAL Cardiac troponin elevation as an evidence of neurogenic myocardial dysfunction

Community Integration Questionnaire ( $P 0.04)$. These findings suggest that the RTS collected in the emergency department is a relevant predictor of outcomes in this population. However, only 120 of 378 eligible patients were available for follow up.

The extension of the RTS to predicting outcome other than mortality in trauma populations is a more recent development and the findings to date have been mixed. Most studies have focused on small discrete populations of head-injured patients ${ }^{(47,51,52)}$, while the one large study of a broad trauma population provided insufficient detail of the measure of morbidity used $\stackrel{(49)}{\text {. No }}$ studies have evaluated the ability of the RTS to predict functional outcome or disability in Australian trauma populations.

Overall, there is substantial evidence to suggest that the RTS collated from observations in the emergency department is a good predictor of mortality following traumatic injury, but there is less evidence to support its use as a predictor of other outcomes such as disability and morbidity.

Finally we can document that our study had two main findings: First, cardiac injury occurs in nearly $18.36 \%$ of patients with TBI. Second, there was an association between cardiac injury and poor outcome in patients with TBI.

Although brain-heart interactions were previously reported in many CNS conditions, only few studies highlighted the link between cardiac injury and TBI (55,56). Most of these mentioned studies enrolled a small series of single cohort of patients with no data about the patients' final outcome.

Our findings have several potential clinical implications in both intensive care and anesthetic practice in cases of TBI. The awareness of the possible presence of cardiac injury in these patients and its impact on patient outcome might raise the attention toward cardiac protection in cases with higher risk. Although there is no consensus till now about the efficacy of any mode of cardiac protection in neurosurgical patients ${ }^{(57)}$, a possible role for propranolol and phentolamine in cardiac protection was suggested by Neil-Dwyer and colleagues (58) who reported the absence of myocardial necrosis in post-mortem specimens for patients with SAH who received these drugs. However, this finding had no impact on patient outcome. The presence of cardiac injury most likely reflects a severe TBI and consequently predicts a poor outcome. It is therefore less likely that the efforts to treat the cardiac condition will influence the outcome.

\section{Conclusion:}

Dexmedetomidine is effective in attenuating the postoperative rise of the proinflammatory cytokine interleukin-6 and tumor necrosis factor (TNF- $\alpha$ ) and resulted in lower levels of markers of stress response to surgery as cortisol and blood glucose.

\section{References:}

1. Babuin L, Jaffe AS. Troponin: the biomarker of choice for detection of cardiac injury. CMAJ. 2005;173:11911202.

2. Roongsritong C, Warraich I, Bradley C. Common causes of troponin elevation in the absence of acute myocardial infarction: incidence and clinical significance. Chest. 2004;125:18771884.

3. Naidech A, Kreiter K, Janjua N, et al. Cardiac troponin elevation, cardiovascular morbidity, and outcome after subarachnoid hemorrhage. Circulation. 2005;112:2851-2856.

4. Hays A, Diringer MN. Elevated troponin levels are associated with higher mortality following intracerebral 
SOHAG MEDICAL JOURNAL Cardiac troponin elevation as an evidence of neurogenic myocardial dysfunction

hemorrhage. Neurology. 2006;66:1330 -1334 .

5. Zaroff JG, Rordorf GA, Ogilvy CS, et al. Regional patterns of left ventricular systolic dysfunction after subarachnoid hemorrhage: evidence for neurally mediated cardiac injury. $J$ Am Soc Echocardiogr. 2000;13:774-779.

6. Schuiling WJ, Dennesen PJ, Tans JT, et al. Troponin I in predicting cardiac or pulmonary complications and outcome in subarachnoid haemorrhage. J Neurol Neurosurg Psychiatry. 2005; 76:15651569.

7. Maramattom BV, Manno EM, Fulgham JR. Clinical importance of cardiac troponin release and cardiac abnormalities in patients with supratentorial cerebral hemorrhages. Mayo Clin Proc. 2006; 81:192-196.

8. Salim A. Significance of Troponin Elevation After Severe Traumatic Brain Injury. J Trauma. 2008;64:46 -52.

9. Salim A, Velmahos GC, Jindal A. Clinically significant blunt cardiac trauma: role of serum troponin levels combined with electrographicfindings. $J$ Trauma. 2001;50:237-243.

10.Lippi G, Dipalo M, Carbucicchio A ,et al. The concentration of highly-sensitive troponin I is increased in patients with brain injury after mild head trauma. International Journal of Cardiology. 2013.

11.Jeremias A, Gibson CM. Narrative review: alternative causes for elevated cardiac troponin levels when acute coronary syndromes are excluded. Ann Intern Med. 2005;142:786 -791.

12. Velmahos GC, Karaiskakis M, Salim A. Normal electrocardiography and serum troponin I levels preclude the presence of clinically significant blunt cardiac injury. J Trauma. 2003;54:45-51.

13. Naidech AM, Kreiter KT, Janjua N, Ostapkovich ND, Parra A, Commichau C, Fitzsimmons BF, Connolly ES, Mayer SA. Cardiac troponin elevation, cardiovascular morbidity, and outcome after subarachnoid hemorrhage. Circulation. 2005;112(18): 2851-6.

14.De'Ath HD, Rourke C, Davenport R, Manson J, Renfrew I, Uppal R, Davies LC, Brohi K. Clinical and biomarker profile of trauma-induced secondary cardiac injury. $\mathrm{Br}$ J Surg. 2012;99(6):789-97.

15.Jeremias A, Gibson CM. Narrative review: alternative causes for elevated cardiac troponin levels when acute coronary syndromes are excluded. Ann Intern Med. 2005;142(9):786-91.

16. Woolf PD, Hamill RW, Lee LA, Cox C, McDonald JV. The predictive value of catecholamines in assessing outcome in traumatic brain injury. J Neurosurg. 1987;66(6):875-82

17.Hamill RW, Woolf PD, McDonald JV, Lee LA, Kelly M. Catecholamines predict outcome in traumatic brain injury. Ann Neurol. 1987;21(5):438-43

18.Clifton GL, Ziegler MG, Grossman RG. Circulating catecholamines and sympathetic activity after head injury. Neurosurgery. 1981;8(1):10-4

19. Alali AS, McCredie VA, Golan E, Shah PS, Nathens AB. Beta blockers for acute traumatic brain injury: a systematic review and meta-analysis. Neurocrit Care. 2014;20(3):514-23

20.Cotton BA, Snodgrass KB, Fleming SB, Carpenter RO, Kemp CD, Arbogast PG, Morris JA., Jr Beta-blocker exposure is associated with improved survival after severe traumatic brain injury. $\mathrm{J}$ Trauma. 2007;62(1):26-33. discussion 33-5.

21.Grunsfeld A, Fletcher JJ, Nathan BR. Cardiopulmonary complications of brain injury. Curr Neurol Neurosci Rep 2005;5:488-93.

22.Chadi Dib, MD,a Samuel Asirvatham, $\mathrm{MD}$, FACC,b Ahmed Elesber, MD,c Charanjit Rihal, MD ,FACC ,b Paul Friedman, MD FACC,b and Abhiram 
SOHAG MEDICAL JOURNAL Cardiac troponin elevation as an evidence of neurogenic myocardial dysfunction

Prasad, MD, FRCP, FESC, FACCb Rochester, $\mathrm{MN}$; and Ashland, KY, 2014.

23.Vijay Krishnamoorthy, Sumidtra Prathep, Deepak Sharma, Edward Gibbons, and Monica S. Vavilala. Association between electrocardiographic findings and cardiac dysfunction in adult isolated traumatic brain injury Indian J Crit care Med. 2014 Sep;18(9):570-574

24.Ahmed Hasanin1*, Amr Kamal1, Shereen Amin1, Dina Zakaria1, Riham El Sayed2, Kareem Mahmoud3 and Ahmed Mukhtar1, Scandinavian Journal of Trauma, Resuscitation and Emergency Medicine (2016) 24:58

25.Prathep S, Sharma D, Hallman M, etal. Preliminary report on cardiac duyfunction after isolated traumatic brain injury.Crit Care Med.2014;42:142-7

26.Serri K, El Rayes M, Giraldeau G, Williamson D, Bernard F. Traumatic braininjury is not associated with significant myocardial dysfunction: anobservational pilot study. Scand J Trauma Resusc Emerg Med. 2016;24:31.

27.James P, Ellis CJ, Whitlock RM, McNeil AR, Henley J, Anderson NE. Relation between troponin $\mathrm{T}$ concentration and mortality in patients presenting with an acute stroke: observational study. BMJ. 2000;320(7248):1502-4.

28.Song HS, Back JH, Jin DK, et al. Cardiac troponin $\mathrm{T}$ elevation after stroke: Relationships between elevated serum troponin $\mathrm{T}$, stroke location, and prognosis. J Clin Neurol. 2008;4:75-83.

29. Coghlan LA, Hindman BJ, Bayman EO. Independent associations between electrocardiographic abnormalities and outcomes in patients with aneurysmal subarachnoid hemorrhage: findings from the intraoperative hypothermia aneurysm surgery trial. Stroke. 2009;40:412-8.
30.Zaroff JG, Leong J, Kim $\mathrm{H}$, et al. Cardiovascular predictors of long-term outcomes after non-traumatic subarachnoid hemorrhage. Neurocrit Care. 2012;17:374-81.

31.Banki N, Kopelnik A, Tung $P$, et al. Prospective analysis of prevalence, distribution, and rate of recovery of left ventricular systolic dysfunction in patients with subarachnoid hemorrhage. J Neurosurg. 2006;105:15-20.

32.Marshall, L.F., Eisenberg, H., Jane, J.A. et al. A new classification of head injury based on computed tomography. $J$ Neurosurg. 1991; 75: S14-S20

33.Maas, A.I., Hukkelhoven, C.W., Marshall, L.F. et al. Prediction of outcome in traumatic brain injury with computed tomographic characteristics: a comparison between the computed tomographic classification and combinations of computed tomographic predictors. (discussion 11731182)Neurosurgery. 2005; 57: 1173 1182

34.Chun, K.A., Manley, G.T., Stiver, S.I. et al. Interobserver variability in the assessment of CT imaging facture of traumatic brain injury. $J$ Neurotrauma. 2010; 27: 325-330

35.Katsnelson, M., Mackenzie, L., Frangos, S. et al. Are initial radiology and clinical scales associated with subsequent intracranial pressure and brain oxygen level after severe traumatic brain injury?.Neurosurgery. 2012; 70: 10951105

36.Huang, Y.H., Deng, Y.H., Lee, T.C. et al. Rotterdam computed tomography score as a prognosticator in head-injured patients undergoing decompressive craniectomy. Neurosurgery. 2012; 71: 8 $0-85$

37.Haydel, M.J., Preston, C.A., Mills, T.J. et al. Indications for computed tomography in patients with minor head injury. $N$ Engl J Med. 2000; 343: 100105 
SOHAG MEDICAL JOURNAL Cardiac troponin elevation as an evidence of neurogenic myocardial dysfunction

38.Stiell, I.G., Lesiuk, H., Wells, G.A. et al. Canadian CT head rule study for patients with minor head injury: methodology for phase II (validation and economic analysis). Ann Emerg Med. 2001; 38: 317-322

39.Stocchetti, N., Croci, M., Spagnoli, D. et al. Mass volume measurement in severe head injury: accuracy and feasibility of two pragmatic methods. $J$ Neurol

Neurosurg

Psychiatry. 2000; 68: 14-17

40.Harrell FE. Regression modeling strategies: with applications to linear models, logistic regression, and survival analysis. Springer series in statistics, Springer-Verlag, New York, Inc, 2001

41.Steyerberg, E.W., Mushkudiani, N., Perel, P. et al. Predicting outcome after traumatic brain injury: development and international validation of prognostic scores based on admission characteristic.PLoS Med. 2008; 5: e165

42.Lingsma, H.F., Roozenbeek, B., Steyerberg, E.W. et al. Early prognosis in traumatic brain injury: from prophecies to predictions. Lancet Neurol. 2010; 9: 543-554

43.Jacobs, B., Beems, T., Stulemeijer, M. et al. Outcome prediction in mild traumatic brain injury: age and clinical variables are stronger predictors than CT abnormalities. $J$

Neurotrauma. 2010; 27: 655-668

44.Gray A, Goyder E, Goodacre S, Johnson G. Trauma triage: a comparison of CRAMS and TRTS in a UK population. Injury 1997; 28: 97-101.

45.Gilpin D, Nelson P. Revised trauma score: a triage tool in the accident and emergency department. Injury 1991; 22: 35-7.

46.Luk SS, Jacobs L, Ciraulo D, Cortes V, Sable A, Dowell VL. Outcome assessment of physiologic and clinical predictors of survival in patients after traumatic injury with a trauma score less than 5. J. Trauma 1999; 46: 122-7.

47. Kilaru S, Garb JL, Emhoff TA et al. Long term functional status and mortality of elderly patients with severe closed head injuries. $J$. Trauma 1996; 41: 957-63

48.Kuhls DA, Malone DL, McCarter RJ, Napolitano LM. Predictors of mortality in adult trauma patients. The physiologic trauma score is equivalent to the trauma and injury severity score. J. Am. Coll. Surg. 2002; 194: 695-704.

49. Moini M, Rezaishiraz H, Zafarghandi MR. Characteristics and outcome of injured patients treated in urban trauma centers in Iran. J. Trauma 2000; 48: 503-7.

50. Brennan PW, Everest ER, Griggs WM et al. Risk of death among cases attending South Australian major trauma services after severe trauma: the first 4 years of operation of a state trauma system. J. Trauma 2002; 53: 333-9

51. Zafonte R, Hammond F, Mann N, Wood D, Millis S, Black K. Revised trauma score: an additive predictor of disability following traumatic brain injury? Am. J. Phys. Med. Rehabil. 1996; 75: 456-61.

52. Wagner AK, Hammond FM, Sasser HC, Wiercisiewski D, Norton HJ. Use of injury severity variables in determining disability and community integration after traumatic brain injury. Trauma J.2000; 49: 411-19

53.Hafiz NA. Evaluation of revised Trauma Score in poly-traumatized patients. Journal of College of Physicians and Surgeons Pakistan. 2004;14(5):286-289

54. Ohaegbulam SC, Okafor UV. Using the Revised Trauma Score to Predict Outcome in Severely Head Injured Patients in a Developing Nation-A Pilot Study. Journal of College of Medicine. 2007;12(2):51-55 
SOHAG MEDICAL JOURNAL Cardiac troponin elevation as an evidence of neurogenic myocardial dysfunction

55.Riera M, Llompart-Pou JA, Carrillo A, Blanco C. Head injury and inverted Takotsubo cardiomyopathy. J Trauma. 2010;68:E13-5.

56. Bahloul BM, Chaari AN, Kallel H, Khabir A, Ayadi A, Charfeddine H, et al. Neurogenic pulmonary edema due to traumatic brain injury: Evidene of cardiac dysfunction. Am J Crit Care. 2006;15:462-70.

57. Okabe T, Kanzaria M, Rincon F, Kraft WK. Cardiovascular protection to improve clinical outcomes after subarachnoid hemorrhage: is there a proven role? Neurocrit Care. 2013;18:271-84.

58.Neil-Dwyer G, Walter P, Cruickshank JM, Doshi B, O'Gorman P. Effect of propranolol and phentolamine on myocardial necrosis after subarachnoid haemorrhage. $\mathrm{Br}$ Med J. 1978;2(6143):990-2. 